\title{
SNR Analysis of Multi-Rate IR-UWB over Frequency-Selective Channels
}

\author{
Henk Wymeersch, Gil Zussman, and Moe Z. Win \\ Massachusetts Institute of Technology, 77 Massachusetts Avenue, Cambridge MA, 02139 \\ e-mail: \{hwymeers,gilz, moewin\}@mit.edu
}

\begin{abstract}
This paper is motivated by the need to design medium access control (MAC) protocols for impulse radio ultrawide bandwidth (UWB) systems. Such protocols should determine how power and data rates should be allocated in order to optimize some performance measure. In order to develop a UWB MAC protocol, there is a need to gain insight on how the signalto-noise ratio (SNR) is affected by different users transmitting with different rates and different power levels. In this paper, we obtain the SNR in a multi-rate system operating in a multi-path environment with time-hopping and/or direct-sequence spreading. We show that the SNR in a time-hopping system is highly affected by the low-rate users.
\end{abstract}

Index Terms - Ultrawide bandwidth (UWB), signal-to-noise ratio (SNR), impulse radio, multi-path, multi-rate, medium access control (MAC).

\section{INTRODUCTION}

Ultrawide bandwidth (UWB) is an emerging technology that has the potential to become the new enabler for low-cost low-power wireless networks. The strength of the UWB radio technique lies in its use of extremely wide transmission bandwidths and very low radiation power, which result in desirable capabilities including accurate location and position ranging, lack of significant fading, and possible material penetration [1]. The 2002 FCC ruling [2] will likely result in the deployment of UWB devices in the near future. Standardization efforts of UWB systems currently take place within the IEEE 802.15.4a task group [3], which aims mainly at ultra-low power applications. A physical layer (PHY) standard for UWB Personal Area Networks has also been developed by the WiMedia Alliance [4].

Specifically, UWB impulse radio (IR) [5] is a physical layer technique that uses a time-hopping baseband signal composed of subnanoseconds pulses (referred to as monocycles) with a very short duty cycle (of the order of one nanosecond). Recent studies have shown that IR-UWB can achieve reliable transmission in challenging environments, with low receiver complexity and power consumption [6]. IR-UWB can operate as the physical layer of ad-hoc and sensor networks, and has several distinct characteristics that set it apart from other narrow-band and wideband technologies. Due to the distinct characteristics of UWB-IR, reusing medium access control (MAC) protocols originally designed for narrow-band systems may be inefficient [7]. Currently, only a few aspects of the
UWB MAC layer have been been studied (e.g. [8], [9]). For example, MAC protocols tailored for the UWB physical layer were proposed in [10]-[13]. In these papers, the authors formulated optimization problems that attempt to capture the particularities of the physical layer and use these problems as a basis for a MAC protocol design. Despite these recent efforts, it is still unclear how power and data rates should be allocated in order to optimize some performance measures, subject to power dissipation and battery consumption constraints.

Most of the optimization approaches build upon the results of [5], regarding multi-user communications in UWB-IR. Interestingly, although the proposed MAC protocols are supposed to allocate different data rates to the different users, [5] considered only a single-rate system, where all the users transmit at the same data rate. Hence, in order to enable an optimization that will lead to a rate allocation protocol, there is a need to obtain results regarding multi-rate systems. In our recent work [14], we have taken the first step in that direction and extended the work of [5] to include multi-rate transmissions over frequencyflat channels. In order to consider a more realistic setting, in this paper, we study UWB multi-path channels. In particular, we consider an UWB system with multi-path channels and multiple transmitters with different power levels and different data rates. We derive lower bounds on the received SNR in the presence of multiple access interference (MAI), for pulseamplitude modulation (PAM) with time-hopping (TH) and/or direct-sequence (DS) signaling.

This paper is organized as follows. In section II we describe the transmission scheme, the channel model, and the receiver. In Section III we derive closed form expressions for the MAI. In Section IV we obtain lower bounds on the SNR and in Section V we summarize the results and discuss future research directions.

\section{SYSTEM MODEL}

\section{A. Transmitters}

We consider a multi-rate version of the UWB-IR system analyzed in [5]. We denote the number of active transmitters (users) by $N_{\mathrm{u}}$ and focus on analyzing the performance of a single receiver ${ }^{1}$. This receiver wishes to recover the data bits

\footnotetext{
${ }^{1}$ When multiple receivers are present, we simple apply our analysis to each of the receivers.
} 


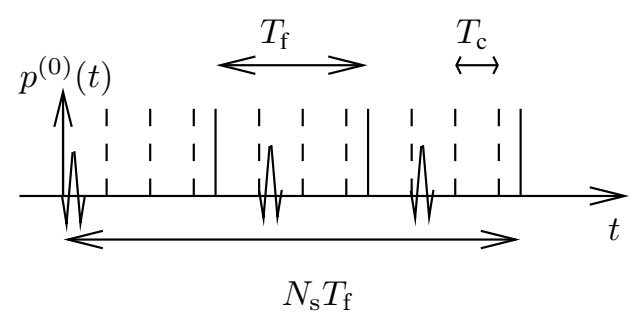

Fig. 1. An example of a transmission by a single user, where $N_{\mathrm{h}}=3$ and $N_{\mathrm{s}}=4$.

from user 0 . The remaining $N_{\mathrm{u}}-1$ users are considered as interferers.

In an UWB-IR system, the transmission of a single data symbol corresponds to sending $N_{\mathrm{s}}$ monocycles over the physical channel and recombining these at the receiver $\left(N_{\mathrm{S}}\right.$ is the processing gain). Each monocycle is of duration $T_{\mathrm{w}}$ (typically less than $1 \mathrm{~ns}$ ) and is transmitted within a chip whose duration is $T_{\mathrm{c}}$. A frame is composed of $N_{\mathrm{h}}$ chips and its length is denoted by $T_{\mathrm{f}}\left(N_{\mathrm{h}} T_{\mathrm{c}}<T_{\mathrm{f}}\right)$. We assume that $T_{\mathrm{c}}$ and $T_{\mathrm{f}}$ are multiples of $T_{\mathrm{w}}$. For each frame, the TH sequence determines the location of the chip in which the transmission takes place. Fig. 1 illustrates an example of a transmission by a single user.

User $k$ transmits a sequence of data symbols (e.g., MPAM symbols), $\left\{a_{l}^{(k)}\right\}_{l=-\infty}^{+\infty}$ with $\mathbb{E}\left\{\left|a_{l}^{(k)}\right|^{2}\right\}=1$ and average transmit power $P_{\mathrm{avg}}^{(k)}$. Since we focus on a multi-rate system we denote the transmission rate of user $k$ by $R^{(k)}$ (symbols per second). Theoretically, the rate on a link can be changed by varying $N_{\mathrm{s}}$ or $T_{\mathrm{f}}$ [15]. We will only consider the more practical case in which different links have different values of $N_{\mathrm{s}}$. Accordingly, we denote the processing gain of user $k$ by $N_{\mathrm{s}}^{(k)}$ and therefore, $R^{(k)}=1 /\left(N_{\mathrm{s}}^{(k)} T_{\mathrm{f}}\right)$. Since we focus on user 0 , we assume that the processing gains of the different users satisfy $N_{\mathrm{s}}^{(k)} / N_{\mathrm{s}}^{(0)} \in \mathbb{N}$ when $N_{\mathrm{s}}^{(k)} \geq N_{\mathrm{s}}^{(0)}$ or $N_{\mathrm{s}}^{(0)} / N_{\mathrm{s}}^{(k)} \in \mathbb{N}$ when $N_{\mathrm{s}}^{(0)} \geq N_{\mathrm{s}}^{(k)}$.

The transmitted waveform from user $k$ is written as,

$$
s^{(k)}(t)=\sqrt{\frac{P_{\mathrm{avg}}^{(k)}}{R^{(k)}}} \sum_{l=-\infty}^{+\infty} a_{l}^{(k)} p^{(k)}\left(t-l T^{(k)}\right)
$$

where $T^{(k)}=1 / R^{(k)}$ and $p^{(k)}(t)$ is the unit-energy transmit pulse for user $k$, given by

$$
p^{(k)}(t)=\frac{1}{\sqrt{N_{\mathrm{s}}^{(k)}}} \sum_{n=0}^{N_{\mathrm{s}}^{(k)}-1} d_{n}^{(k)} w\left(t-c_{n}^{(k)} T_{\mathrm{c}}-n T_{\mathrm{f}}\right) .
$$

Above, $w(t)$ denotes the monocycle, $d_{n}^{(k)} \in\{-1,1\}$ represents a DS and $c_{n}^{(k)} \in\left\{0,1, \ldots, N_{\mathrm{h}}-1\right\}$ represents a TH sequence. For a pure TH system, $d_{n}^{(k)}=1, \forall n, k$. We assume that the $c_{n}^{(k)}$, s are uniformly (and independently) distributed in the set $\left\{0,1, \ldots, N_{\mathrm{h}}-1\right\} \forall n, k$.

\section{B. Channel Model}

The signal $s^{(k)}(t)$ propagates through the physical channel $h^{(k)}(t)$ between user $k$ and the receiver. We will consider a frequency-selective channel of the form

$$
h^{(k)}(t)=\sum_{i=0}^{L-1} \alpha_{i}^{(k)} \delta\left(t-\tau^{(k)}-i T_{\mathrm{w}}\right)
$$

where $\tau^{(k)}$ represents the timing offset between the $k$-th user and the receiver, $L$ is the maximum number of resolvable multipath components ${ }^{2}$, and $\alpha_{i}^{(k)}$ is the (complex) attenuation of the $i$-th path. Note that $L T_{\mathrm{w}}$ is equal to the maximal delay spread of the channel.

\section{Receiver}

Ignoring antenna effects, the signal at the input of the receiver is given by

$$
\begin{aligned}
r(t) & =\sum_{k=0}^{N_{\mathrm{u}}-1} \sum_{i=0}^{L-1} \alpha_{i}^{(k)} s^{(k)}\left(t-\tau^{(k)}-i T_{\mathrm{w}}\right)+v(t) \\
& =\sum_{k=0}^{N_{\mathrm{u}}-1} \sqrt{\frac{P_{\mathrm{avg}}^{(k)}}{R^{(k)}}} \sum_{l=-\infty}^{+\infty} a_{l}^{(k)} q^{(k)}\left(t-l T^{(k)}\right)+v(t)
\end{aligned}
$$

where $v(t)$ is a complex wideband AWGN process with psd $N_{0} / 2$ for both the real and imaginary part, and

$$
q^{(k)}(t)=\sum_{i=0}^{L-1} \alpha_{i}^{(k)} p^{(k)}\left(t-\tau^{(k)}-i T_{\mathrm{w}}\right) .
$$

Recall that the receiver is only interested in recovering the data symbols $\left\{a_{l}^{(0)}\right\}_{l=-\infty}^{+\infty}$ from user 0 , while the $N_{\mathrm{u}}-1$ remaining users are considered interferers. In order to make a decision with respect to symbol $a_{m}^{(0)}$, the received signal $r(t)$ is filtered by $\left(q^{(0)}(-t)\right)^{*}$ and sampled at time $t=m T^{(0)}$. This results in the decision statistic

$$
z_{m}=\underbrace{\sqrt{\frac{P_{\mathrm{avg}}^{(0)}}{R^{(0)}}} \phi_{m}^{(0)}}_{\text {useful part }}+\underbrace{\sum_{k=1}^{N_{\mathrm{u}}-1} \sqrt{\frac{P_{\mathrm{avg}}^{(k)}}{R^{(k)}}} \phi_{m}^{(k)}}_{\text {MAI }}+n_{m}
$$

where $n_{m}$ is an i.i.d. random variable with variance $N_{0} / 2$ per real dimension. Closed-form expressions for $\phi_{m}^{(k)}$ will be given in section IV. Our goal is to evaluate the SNR, given by

$$
\mathrm{SNR}^{(0)}=\frac{\mathbb{E}\left\{\left|\sqrt{\frac{P_{\text {avg }}^{(0)}}{R^{(0)}}} \phi_{m}^{(0)}\right|^{2}\right\}}{\mathbb{E}\left\{\left|\sum_{k=1}^{N_{\mathrm{u}}-1} \sqrt{\frac{P_{\text {avg }}^{(k)}}{R^{(k)}}} \phi_{m}^{(k)}\right|^{2}\right\}+\mathbb{E}\left\{\left|n_{m}\right|^{2}\right\}} .
$$

The expectations are taken with respect to the noise, the data symbols, and the DS and TH sequences, but for a fixed realization of the channels $h^{(k)}(t)$. Note that since $\mathrm{SNR}^{(0)}$ does not depend on the time index $m$, we can set $m=0$ without

\footnotetext{
${ }^{2} L$ is maximized over all users.
} 
any loss of generality. It is easily verified that $\mathbb{E}\left\{\phi_{0}^{(k)}\right\}=0$ and that, due to the independence of the different transmitters, the multiple access interference (MAI) can be written as

$$
\mathbb{E}\left\{\left|\sum_{k=1}^{N_{\mathrm{u}}-1} \sqrt{\frac{P_{\text {avg }}^{(k)}}{R^{(k)}}} \phi_{0}^{(k)}\right|^{2}\right\}=\sum_{k=1}^{N_{\mathrm{u}}-1} \frac{P_{\text {avg }}^{(k)}}{R^{(k)}} \mathbb{E}\left\{\left|\phi_{0}^{(k)}\right|^{2}\right\} .
$$

\section{Characterization OF THE MAI}

In this section we will derive closed-form expressions of the MAI. It will turn out that we will need to discern between users with a rate lower than user 0 , and users with a rate higher than user 0 . Higher-rate users will contribute less to the MAI, due to the averaging effect of their high-rate transmission. Moreover, we will obtain different results for systems with DS/TH and pure TH systems.

\section{A. General Expressions}

Evaluating the MAI is in general quite cumbersome. We propose to upper bound the MAI (and hence lower bound the SNR) by considering a synchronized scenario whereby $\tau^{(k)}=\tau^{(0)}, \forall k$. This allows us to express $\phi_{0}^{(k)}$ as

$$
\begin{aligned}
\phi_{0}^{(k)} & =\sum_{l=-\infty}^{+\infty} a_{l}^{(k)} \int_{-\infty}^{+\infty} q^{(k)}\left(t-l T^{(k)}\right)\left(q^{(0)}(t)\right)^{*} d t \\
& =\sum_{l=-\infty}^{+\infty} a_{l}^{(k)} \sum_{i, i^{\prime}=0}^{L-1} \alpha_{i}^{(k)}\left(\alpha_{i^{\prime}}^{(0)}\right)^{*} \rho_{i^{\prime}, i}^{(0, k)}(l)
\end{aligned}
$$

where

$$
\rho_{i^{\prime}, i}^{(0, k)}(l)=\int_{-\infty}^{+\infty} p^{(k)}\left(t-i T_{\mathrm{w}}-l T^{(k)}\right)\left(p^{(0)}\left(t-i^{\prime} T_{\mathrm{w}}\right)\right)^{*} d t .
$$

Now, due to (1), $\rho_{i^{\prime}, i}^{(0, k)}(l)$ can be further expanded as

$$
\begin{aligned}
& \rho_{i^{\prime}, i}^{(0, k)}(l)= \\
& \frac{1}{\sqrt{N_{\mathrm{s}}^{(0)} N_{\mathrm{s}}^{(k)}}} \sum_{n=0}^{N_{\mathrm{s}}^{(k)}-1} \sum_{n^{\prime}=0}^{N_{\mathrm{s}}^{(0)}-1} d_{n}^{(k)}\left(d_{n^{\prime}}^{(0)}\right)^{*} \xi_{i^{\prime}, i, n^{\prime}, n}^{(0, k)}(l)
\end{aligned}
$$

with

$$
\begin{aligned}
\xi_{i^{\prime}, i, n^{\prime}, n}^{(0, k)}(l)=\int_{-\infty}^{+\infty}\left\{w\left(t-c_{n}^{(k)} T_{\mathrm{c}}-n T_{\mathrm{f}}-i T_{\mathrm{w}}-l T^{(k)}\right)\right. \\
\left.\times w\left(t-c_{n^{\prime}}^{(0)} T_{\mathrm{c}}-n^{\prime} T_{\mathrm{f}}-i^{\prime} T_{\mathrm{w}}\right)\right\} d t .
\end{aligned}
$$

Since the duration of a monocycle $w(t)$ is exactly $T_{\mathrm{w}}$, and monocycles are of unit energy, $\xi_{i^{\prime}, i, n^{\prime}, n}^{(0, k)}(l)=1$ when

$$
c_{n}^{(k)} T_{\mathrm{c}}+n T_{\mathrm{f}}+i T_{\mathrm{w}}+l T^{(k)}=c_{n^{\prime}}^{(0)} T_{\mathrm{c}}+n^{\prime} T_{\mathrm{f}}+i^{\prime} T_{\mathrm{w}}
$$

and $\xi_{i^{\prime}, i, n^{\prime}, n}^{(0, k)}(l)=0$, otherwise. We assume the UWB system has been designed such that there is no inter-frame interference (i.e., that $\left.\left(N_{h}-1\right) T_{\mathrm{c}}+(L-1) T_{\mathrm{w}}<T_{\mathrm{f}}\right)$, and hence no intersymbol interference (i.e., $\left(N_{h}-1\right) T_{\mathrm{c}}+(L-1) T_{\mathrm{w}}+\left(N_{s}^{(k)}-\right.$ 1) $\left.T_{\mathrm{f}}<T^{(k)} \forall k\right)$.
Now, when $T^{(k)} \geq T^{(0)}$, we find that only $l=0$ can make $\xi_{i^{\prime}, i, n^{\prime}, n}^{(0, k)}(l) \neq 0$. On the other hand, when $T^{(k)}<T^{(0)}$, multiple values of $l$ may give rise to $\xi_{i^{\prime}, i, n^{\prime}, n}^{(0, k)}(l) \neq 0$. Hence, we need to discern two cases: the case of users with a rate lower than or equal to user 0 , and users with a rate higher than user 0 .

\section{B. Case 1: Users with a rate lower than (or equal to) user 0}

When $R^{(k)} \leq R^{(0)}, T^{(k)} \geq T^{(0)}$. In such a case, $l$ must be 0 in order to have $\xi_{i^{\prime}, i, n^{\prime}, n}^{(0, k)}(l) \neq 0$. Furthermore, since $\left(N_{\mathrm{h}}-1\right) T_{\mathrm{c}}+(L-1) T_{\mathrm{w}}<T_{\mathrm{f}}$, we find that $n=n^{\prime}$, so that $\xi_{i^{\prime}, i, n, n}^{(0, k)}(0)=1$ if and only if

$$
c_{n}^{(k)} T_{\mathrm{c}}+i T_{\mathrm{w}}=c_{n}^{(0)} T_{\mathrm{c}}+i^{\prime} T_{\mathrm{w}} .
$$

Let us introduce the random variable $\psi_{n}=\left(c_{n}^{(0)}-c_{n}^{(k)}\right) T_{\mathrm{c}} / T_{\mathrm{w}}$. Since $c_{n}^{(0)}$ and $c_{n}^{(k)}$ are uniformly (and independently) distributed in the set $\left\{0,1, \ldots, N_{\mathrm{h}}-1\right\}$, we find the distribution of $\psi_{n}$ to be as follows:

$$
\mathbb{P}\left\{\psi_{n}=g \frac{T_{\mathrm{c}}}{T_{\mathrm{w}}}\right\}=\frac{N_{\mathrm{h}}-|g|}{N_{\mathrm{h}}^{2}}
$$

for $g \in\left\{0, \pm 1, \pm 2, \ldots, \pm\left(N_{\mathrm{h}}-1\right)\right\}$. Note that given values of $\psi_{n}$ and $i, i^{\prime}=i-\psi_{n}$ has to hold in order to satisfy (3).

Finally, we can express $\phi_{0}^{(k)}$ as

$$
\begin{aligned}
& \phi_{0}^{(k)}= \\
& \frac{1}{\sqrt{N_{\mathrm{s}}^{(0)} N_{\mathrm{s}}^{(k)}}} a_{0}^{(k)} \sum_{n=0}^{N_{\mathrm{s}}^{(0)}-1} d_{n}^{(k)}\left(d_{n}^{(0)}\right)^{*} \sum_{i=0}^{L-1} \alpha_{i}^{(k)}\left(\alpha_{i-\psi_{n}}^{(0)}\right)^{*} .
\end{aligned}
$$

By definition $0 \leq i, i-\psi_{n}<L$. Therefore, as long as $0 \leq i-$ $\psi_{n}<L$, multiple values of $\psi_{n}$ can lead to $\xi_{i-\psi_{n}, i, n, n}^{(0, k)}(0) \neq 0$. For instance, when $L<2 T_{\mathrm{c}} / T_{\mathrm{w}}$, values of $\psi_{n}$ such that $\psi_{n} \in$ $\left\{-\frac{T_{\mathrm{c}}}{T_{\mathrm{w}}}, 0, \frac{T_{\mathrm{c}}}{T_{\mathrm{w}}}\right\}$ are possible. For simplicity of the exposition, we will assume $L<T_{\mathrm{c}} / T_{\mathrm{w}}$, so that only $\psi_{n}=0$ can lead to $\xi_{i-\psi_{n}, i, n, n}^{(0, k)}(0) \neq 0$, in a sense that if $\psi_{n} \neq 0$, it necessarily follows that $\xi_{i-\psi_{n}, i, n, n}^{(0, k)}(0)=0$. The extension to longer delay spreads is straightforward, and does not give any additional insight.

We are now ready to compute $\mathbb{E}\left\{\left|\phi_{0}^{(k)}\right|^{2}\right\}$, but must make a distinction between a combined DS/TH system, and a pure TH system (in the latter $d_{n}^{(k)} \equiv 1$ ).

Case 1.1 Joint DS/TH system: For a system with DS/TH, we find that

$$
\begin{aligned}
& \mathbb{E}\left\{\left|\phi_{0}^{(k)}\right|^{2}\right\}=\frac{1}{N_{\mathrm{s}}^{(0)} N_{\mathrm{s}}^{(k)}} \sum_{n, u=0}^{N_{\mathrm{s}}^{(0)}-1} \sum_{i, j=0}^{L-1} \alpha_{i}^{(k)}\left(\alpha_{j}^{(k)}\right)^{*} \\
& \times \mathbb{E}\left\{d_{n}^{(k)}\left(d_{u}^{(k)}\right)^{*}\left(d_{n}^{(0)}\right)^{*} d_{u}^{(0)}\left(\alpha_{i-\psi_{n}}^{(0)}\right)^{*} \alpha_{j-\psi_{u}}^{(0)}\right\} .
\end{aligned}
$$


Since the DS of the different users are independent, and since $\mathbb{E}\left\{d_{n}^{(k)}\left(d_{u}^{(k)}\right)^{*}\right\}=\delta_{n-u}$, we find that

$$
\begin{aligned}
& \mathbb{E}\left\{\left|\phi_{0}^{(k)}\right|^{2}\right\}= \\
& \frac{1}{N_{\mathrm{s}}^{(0)} N_{\mathrm{s}}^{(k)}} \sum_{n=0}^{N_{\mathrm{s}}^{(0)}-1} \sum_{i, j=0}^{L-1} \alpha_{i}^{(k)}\left(\alpha_{j}^{(k)}\right)^{*} \mathbb{E}\left\{\left(\alpha_{i-\psi_{n}}^{(0)}\right)^{*} \alpha_{j-\psi_{n}}^{(0)}\right\} .
\end{aligned}
$$

where the expectation is now only over the random variable $\psi_{n}$. Since only $\psi_{n}=0$ contributes to the MAI,

$$
\begin{aligned}
\mathbb{E}\left\{\left(\alpha_{i-\psi_{n}}^{(0)}\right)^{*} \alpha_{j-\psi_{n}}^{(0)}\right\} & =P\left\{\psi_{n}=0\right\}\left(\alpha_{i}^{(0)}\right)^{*} \alpha_{j}^{(0)} \\
& =\frac{1}{N_{\mathrm{h}}}\left(\alpha_{i}^{(0)}\right)^{*} \alpha_{j}^{(0)}
\end{aligned}
$$

We now define

$$
\eta^{(k)}=\left|\sum_{i=0}^{L-1} \alpha_{i}^{(k)}\left(\alpha_{i}^{(0)}\right)\right|^{2}
$$

and get

$$
\mathbb{E}\left\{\left|\phi_{0}^{(k)}\right|^{2}\right\}=\frac{\eta^{(k)}}{N_{\mathrm{s}}^{(k)} N_{\mathrm{h}}} .
$$

Case 1.2 TH system: For a system without DS, $d_{n}^{(k)}=1$, so that

$$
\begin{aligned}
& \mathbb{E}\left\{\left|\phi_{0}^{(k)}\right|^{2}\right\} \\
& =\frac{1}{N_{\mathrm{s}}^{(0)} N_{\mathrm{s}}^{(k)}} \sum_{n, u=0}^{N_{\mathrm{s}}^{(0)}} \sum_{i, j=0}^{L-1} \alpha_{i}^{(k)}\left(\alpha_{j}^{(k)}\right)^{*} \mathbb{E}\left\{\left(\alpha_{i-\psi_{n}}^{(0)}\right)^{*} \alpha_{j-\psi_{u}}^{(0)}\right\} \\
& =\frac{1}{N_{\mathrm{s}}^{(0)} N_{\mathrm{s}}^{(k)}} \sum_{n=0}^{N_{\mathrm{s}}^{(0)}-1} \sum_{i, j=0}^{L-1} \alpha_{i}^{(k)}\left(\alpha_{j}^{(k)}\right)^{*} \mathbb{E}\left\{\left(\alpha_{i-\psi_{n}}^{(0)}\right)^{*} \alpha_{j-\psi_{n}}^{(0)}\right\} \\
& +\frac{1}{N_{\mathrm{s}}^{(0)} N_{\mathrm{s}}^{(k)}} \sum_{n=0}^{N_{\mathrm{s}}^{(0)}-1} \sum_{\substack{u=0 \\
u \neq n}}^{N_{\mathrm{s}}^{(0)}-1} \sum_{i, j=0}^{L-1} \alpha_{i}^{(k)}\left(\alpha_{j}^{(k)}\right)^{*} \\
& \times \mathbb{E}\left\{\left(\alpha_{i-\psi_{n}}^{(0)}\right)^{*} \alpha_{j-\psi_{u}}^{(0)}\right\} .
\end{aligned}
$$

Now the first term is the same as in the DS/TH case, and thus equal to $\eta^{(k)} /\left(N_{\mathrm{S}}^{(k)} N_{\mathrm{h}}\right)$ while the second term is additional MAI, equal to

$$
\begin{aligned}
& \frac{1}{N_{\mathrm{s}}^{(0)} N_{\mathrm{s}}^{(k)}} \sum_{n=0}^{N_{\mathrm{s}}^{(0)}-1} \sum_{\substack{u=0 \\
u \neq n}}^{N_{\mathrm{s}}^{(0)}-1} \sum_{i, j=0}^{L-1} \alpha_{i}^{(k)}\left(\alpha_{j}^{(k)}\right)^{*} \frac{1}{N_{\mathrm{h}}^{2}}\left(\alpha_{i}^{(0)}\right)^{*} \alpha_{j}^{(0)} \\
& =\frac{\eta^{(k)}\left(N_{\mathrm{s}}^{(0)}-1\right)}{N_{\mathrm{s}}^{(k)} N_{\mathrm{h}}^{2}} .
\end{aligned}
$$

All this leads to

$$
\mathbb{E}\left\{\left|\phi_{0}^{(k)}\right|^{2}\right\}=\frac{\eta^{(k)}}{N_{\mathrm{s}}^{(k)} N_{\mathrm{h}}}\left\{1+\frac{\left(N_{\mathrm{s}}^{(0)}-1\right)}{N_{\mathrm{h}}}\right\} .
$$

C. Case 2: Users with a rate higher than user 0

Recall that $\xi_{i^{\prime}, i, n^{\prime}, n}^{(0, k)}(l)=1$ when

$$
c_{n}^{(k)} T_{\mathrm{c}}+n T_{\mathrm{f}}+i T_{\mathrm{w}}+l T^{(k)}=c_{n^{\prime}}^{(0)} T_{\mathrm{c}}+n^{\prime} T_{\mathrm{f}}+i^{\prime} T_{\mathrm{w}}
$$

and $\xi_{i^{\prime}, i, n^{\prime}, n}^{(0, k)}(l)=0$, otherwise. Since the rate of user $k$ is higher that the rate of user $0, T^{(0)} / T^{(k)} \in \mathbb{N}$. This implies that during the time interval $\left[0, T^{(0)}\right]$, exactly $R^{(k)} / R^{(0)}$ symbols of user $k$ cause interference (i.e., $a_{0}^{(k)}, a_{1}^{(k)}, \ldots a_{R^{(k)} / R^{(0)}-1}^{(k)}$ ). This means that $l \in\left\{0,1, \ldots, R^{(k)} / R^{(0)}-1\right\}$ can lead to $\xi_{i^{\prime}, i, n^{\prime}, n}^{(0, k)}(l)=1$. For a fixed $l$ and $n$, we find that $n^{\prime}=$ $n+l T^{(k)} / T_{\mathrm{f}}$, and that $c_{n}^{(k)} T_{\mathrm{c}}+i T_{\mathrm{w}}=c_{n+l T^{(k)} / T_{\mathrm{f}}}^{(0)} T_{\mathrm{c}}+i^{\prime} T_{\mathrm{w}}$

$$
i^{\prime}=i-\underbrace{\left(c_{n+l T^{(k)} / T_{\mathrm{f}}}^{(0)}-c_{n}^{(k)}\right) \frac{T_{\mathrm{c}}}{T_{\mathrm{w}}}}_{\psi_{n, l}} .
$$

The distribution of $\psi_{n, l}$ is exactly the same as of $\psi_{n}$ from the previous section. This leads to

$$
\begin{aligned}
& \phi_{0}^{(k)}=\frac{1}{\sqrt{N_{\mathrm{s}}^{(0)} N_{\mathrm{s}}^{(k)}}} \sum_{l=0}^{R^{(k)} / R^{(0)}-1} a_{l}^{(k)} \\
& \times \sum_{n=0}^{N_{\mathrm{s}}^{(k)}-1} d_{n}^{(k)}\left(d_{n+l T^{(k)} / T_{\mathrm{f}}}^{(0)}\right)^{*} \sum_{i=0}^{L-1} \alpha_{i}^{(k)}\left(\alpha_{i-\psi_{n, l}}^{(0)}\right)^{*} .
\end{aligned}
$$

As before, we will assume $L<T_{\mathrm{c}} / T_{\mathrm{w}}$, so that only $\psi_{n, l}=$ 0 can lead to $\xi_{i-\psi_{n, l}, i, n+l T^{(k)} / T_{\mathrm{f}}, n}^{(0, k)}(l) \neq 0$. The extension to longer delay spreads is straightforward, and does not give any additional insight.

Case 2.1 Joint DS/TH system: For a system with DS/TH, after some manipulations, we find that

$$
\mathbb{E}\left\{\left|\phi_{0}^{(k)}\right|^{2}\right\}=\frac{\eta^{(k)}}{N_{\mathrm{s}}^{(k)} N_{\mathrm{h}}} .
$$

Case 2.2: TH system: For a system without DS, $d_{n}^{(k)}=1$. After some straightforward manipulations we find that

$$
\mathbb{E}\left\{\left|\phi_{0}^{(k)}\right|^{2}\right\}=\frac{\eta^{(k)}}{N_{\mathrm{s}}^{(k)} N_{\mathrm{h}}}\left\{1+\frac{\left(N_{\mathrm{s}}^{(k)}-1\right)}{N_{\mathrm{h}}}\right\} .
$$

\section{SNR EVALUATION}

We can now obtain a lower bound on the SNR. Let us introduce $S_{\mathrm{L}}^{(0)} \subset\left\{1, \ldots, N_{\mathrm{u}}-1\right\}$ as the set of users with a rate lower than (or equal to) user 0 , and $S_{\mathrm{H}}^{(0)}$ as the set of users with a rate higher than user 0 .

\section{A. Contribution from noise and user 0}

It is easily seen that

$$
\phi_{0}^{(0)}=a_{0}^{(0)} \sum_{i, i^{\prime}=0}^{L-1} \alpha_{i}^{(0)}\left(\alpha_{i^{\prime}}^{(0)}\right)^{*} \rho_{i^{\prime}, i}^{(0,0)}(0) .
$$




$$
\begin{aligned}
\mathrm{SNR}^{(0)} & =\frac{P_{\mathrm{avg}}^{(0)} \eta^{(0)}}{R^{(0)}}\left\{N_{0} \sqrt{\eta^{(0)}}+\frac{T_{\mathrm{f}}}{N_{\mathrm{h}}}\left\{\sum_{k \in S_{\mathrm{L}}^{(0)}} P_{\mathrm{avg}}^{(k)} \eta^{(k)}\left(1+\frac{\left(N_{\mathrm{s}}^{(0)}-1\right)}{N_{\mathrm{h}}}\right)+\sum_{k \in S_{\mathrm{H}}^{(0)}} P_{\mathrm{avg}}^{(k)} \eta^{(k)}\left(1+\frac{\left(N_{\mathrm{s}}^{(k)}-1\right)}{N_{\mathrm{h}}}\right)\right\}\right\}^{-1} \\
& \approx \frac{P_{\mathrm{avg}}^{(0)} \eta^{(0)}}{R^{(0)}}\left\{N_{0} \sqrt{\eta^{(0)}}+\sum_{k \in S_{\mathrm{L}}^{(0)}} P_{\mathrm{avg}}^{(k)} \eta^{(k)}\left(\frac{T_{\mathrm{f}}}{N_{\mathrm{h}}}+\frac{1}{R^{(0)} N_{\mathrm{h}}^{2}}\right)+\sum_{k \in S_{\mathrm{H}}^{(0)}} P_{\mathrm{avg}}^{(k)} \eta^{(k)}\left(\frac{T_{\mathrm{f}}}{N_{\mathrm{h}}}+\frac{1}{R^{(k)} N_{\mathrm{h}}^{2}}\right)\right\}
\end{aligned}
$$

Since $\rho_{i^{\prime}, i}^{(0,0)}(0)=\delta_{i-i^{\prime}}, \phi_{0}^{(0)}=a_{0}^{(0)} \sum_{i=0}^{L-1}\left|\alpha_{i}^{(0)}\right|$, so that using the definition of $\eta^{(k)}$ from (4) we get

$$
\mathbb{E}\left\{\left|\phi_{0}^{(0)}\right|^{2}\right\}=\left.\left.\left|\sum_{i=0}^{L-1}\right| \alpha_{i}^{(0)}\right|^{2}\right|^{2}=\eta^{(0)} .
$$

As far as the noise is concerned, $n_{0}=$ $\int_{-\infty}^{+\infty} v(t)\left(q^{(0)}(t)\right)^{*} d t$, so that, since $\mathbb{E}\left\{v(t) v^{*}(u)\right\}=$ $N_{0}^{\infty} \delta(t-u)$,

$$
\mathbb{E}\left\{\left|n_{0}\right|^{2}\right\}=N_{0} \sum_{i=0}^{L-1}\left|\alpha_{i}^{(0)}\right|^{2}=N_{0} \sqrt{\eta^{(0)}} .
$$

\section{B. SNR expressions for a TH/DS system}

For a DS/TH system, low-rate users and high-rate users should cause exactly the same amount of interference. Indeed, for any user $k$, we see that

$$
\frac{P_{\mathrm{avg}}^{(k)}}{R^{(k)}} \mathbb{E}\left\{\left|\phi_{0}^{(k)}\right|^{2}\right\}=\frac{P_{\mathrm{avg}}^{(k)}}{R^{(k)}} \frac{\eta^{(k)}}{N_{\mathrm{s}}^{(k)} N_{\mathrm{h}}}=P_{\mathrm{avg}}^{(k)} \eta^{(k)} \frac{T_{\mathrm{f}}}{N_{\mathrm{h}}}
$$

which is independent of the specific rate of user $k$. Substitution of (8), (9) and (10) into (2) yields the following:

$$
\mathrm{SNR}^{(0)}=\frac{P_{\mathrm{avg}}^{(0)} \eta^{(0)}}{R^{(0)}}\left\{N_{0} \sqrt{\eta^{(0)}}+\frac{T_{\mathrm{f}}}{N_{\mathrm{h}}} \sum_{k=1}^{N_{\mathrm{u}}-1} P_{\mathrm{avg}}^{(k)} \eta^{(k)}\right\}^{-1} .
$$

Clearly, for a frequency-flat channel with $L=1$, (11) reverts to the corresponding expression (eq. (9)) in [14].

\section{SNR expressions for a TH system}

When there is no DS, we need to make a distinction between low-rate users and high-rate users. Substitution of (6), (7), (8), and (9) into (2) yields eq. (12), above. It can be seen that for $L=1$, (12) reverts to the corresponding expression (eq. (8)) in [14].

We see that for a low-rate user, its contribution to the MAI does not depend on its rate, while for a high-rate user, the higher the rate, the less that user causes MAI.

\section{CONCLUSIONS}

Motivated by the need to design MAC protocols for multirate IR-UWB networks, we obtained lower bounds on the SNR in multi-rate UWB-IR systems in multi-path environments. The SNR equations differ from previous results and can form the basis of optimization problems that provide insight into how rate and power should be allocated in UWB-IR networks. Our work can be extended to other multi-rate techniques: apart from changing the data rate by varying the frame duration $T_{\mathrm{f}}$ (which is of little practical importance), extension to a multicode multi-rate scheme [16] is achieved by treating the different data streams of a user as coming from virtual users. Extensions to include the (possibly user-dependent) code rate of an errorcorrecting code are straightforward. Extension of this work to an asynchronous multi-path channel remains a topic for further research.

\section{ACKNOWLEDGMENTS}

This work was supported in part by a Belgian American Educational Foundation fellowship, a Marie Curie Fellowship within the 6th EC Framework Programme, the MAGNET/ISRC Consortium, ONR Young Investigator Award N00014-03-10489 and Grant N000140610064, NSF Grants ANI-0335256 and ECS-0636519, and the Draper Laboratory Robust Distributed Sensor Networks Program.

\section{REFERENCES}

[1] D. Porcino and W. Hirt, "Ultra-Wideband Radio Technology: Potential and Challenges Ahead," IEEE Comm., Vol. 41, pp. 66-74, July 2003.

[2] Federal Communications Commission (FCC), "Revision of Part 15 of the Commission's Rules Regarding Ultra-Wideband Transmission Systems", First Report and Order, ET Docket 98-153, FCC 02-48; Adopted: February 14,2002; Released: April 22, 2002.

[3] IEEE 802.15.4a/D5, "Draft Amendment to IEEE Standard for Information technology-Telecommunications and information exchange between systems-PART 15.4:Wireless Medium Access Control (MAC) and Physical Layer (PHY) Specifications for Low-Rate Wireless Personal Area Networks (LR-WPANs): Amendment to add alternate PHY (Amendment of IEEE Std 802.15.4)", IEEE, Sep 2006.

[4] J. del Prado Pavon, S. Shankar N, V. Gaddam, K. Challapali, C.-T. Chou, "The MBOA-WiMedia specification for ultra wideband distributed networks", IEEE Comm., Vol. 44, No. 6, pp. 128-134, June 2006.

[5] M. Z. Win and R. A. Scholtz., "Ultra-wide bandwidth time-hopping spread-spectrum impulse radio for wireless multiple-access communications," IEEE Trans. on Comm., Vol. 48, No. 4, pp. 679-691, Apr. 2000.

[6] M.-G. Di Benedetto, T. Kaiser, A.F. Molisch, I. Opperman, C. Politano and D. Porcino, UWB Communication Systems: A Comprehensive Overview, Eurasip-Hindawi, 2006.

[7] B. Radunovic and J. Y. Le Boudec, "Optimal power control, scheduling and routing in UWB networks," IEEE JSAC, Vol. 22, No. 7, pp. 12521270, Sep. 2004.

[8] M.-G. Di Benedetto, L. De Nardis, M. Junk, and G. Giancola, "(UWB $)^{2}$. uncoordinated, wireless, baseborn, medium access control for UWB communication networks," ACM/Springer MONET, Vol. 10, No. 5, pp. $663-$ 674, 2005.

[9] R. Merz, J. Widmer, J.-Y. Le Boudec and B. Radunovic, "A joint PHY/MAC architecture for low-radiated power TH-UWB wireless ad-hoc networks," Wireless Comm. and Mobile Comp., Vol. 5, No. 5, pp. $567-$ 580, 2005. 
[10] Y. Chu and A. Ganz, "A centralized MAC protocol for QoS support in UWB-based wireless networks," Wireless Personal Comm., Vol. 34, No. 1-2, pp. 45-66, July 2005.

[11] F. Cuomo, C. Martello, A. Baiocchi, and C. Fabrizio, "Radio resource sharing for ad hoc networking with UWB," IEEE JSAC, Vol. 20, No. 9, pp. 1722-1732, Dec. 2002.

[12] G. Giancola, C. Martello, F. Cuomo, and M.-G. Di Benedetto, "Radio resource management in infrastructure-based and ad hoc UWB networks," Wireless Comm. and Mobile Comp., Vol. 5, No. 5, pp. 581-597, 2005.

[13] R. Jurdak, P. Baldi, C. V. Lopes, "U-MAC: a proactive and adaptive UWB medium access control protocol," Wireless Comm. and Mobile Comp., Vol. 5, No. 5, pp. 551-566, 2005.

[14] H. Wymeersch, G. Zussman, and M. Z. Win, "SNR Analysis for MultiRate UWB-IR," IEEE Comm. Lett., Vol. 11, No. 1, pp. 49-51, Jan. 2007.

[15] H. Wymeersch and M. Moeneclaey, "ML rate detection for multi-rate THUWB impulse radio," in Proc. IEEE ICU 2005, pp. 391-395, Sep. 2005.

[16] M. Nasiri-Kenari and M. G. Shayesteh, "Performance analysis and comparison of different multirate TH-UWB systems: uncoded and coded schemes," IEE Proc.-Comm., Vol. 152, No. 6, pp. 833-844, Dec. 2005. 